\title{
Editorial
}

\section{Esmolol - just another beta blocker?}

In this issue of the Journal, Chung et al. report the results of a double-blind randomized trial designed to determine the efficacy of a bolus dose of fentanyl or esmolol alone, or a combination of low-dose fentanyl and esmolol, in blunting the response to laryngoscopy and tracheal intubation. ' Their report is timely since esmolol (Brevibloc®) has recently been released for clinical use in Canada and anaesthetists will be asked to consider using this agent in their clinical practice.

The mandate for most Pharmacy and Therapeutics (P\&T) Committees is to regulate the introduction of new therapeutic agents into the hospital prescribing environment, to determine that they have proven efficacy, and monitor the ever-increasing costs of therapeutic agents. Under current budgetary constraints, increasing drug costs due to addition of new therapeutic agents to the hospital formulary may mean a reduction in services to patients in some other area, e.g., staff layoffs, bed closures, reductions in operating room availability, etc. Under these circumstances, the addition of new agents to the formulary can only be justified if they provide an alternative to patient management not previously available or have some unique feature which separates them from a whole group of other drugs with otherwise similar characteristics. Esmolol will be considered under the latter category since there are currently seven beta adrenergic receptor blocking agents (BB) available in Canada. ${ }^{2}$

Esmolol is available for intravenous administration only. ${ }^{3}$ Only two other BB - propranolol and metoprolol are available for parenteral administration. ${ }^{2}$ The product is available in three strengths: (1) $10 \mathrm{mg} \cdot \mathrm{ml}^{-1}$ vials for direct injection, and (2) $100 \mathrm{mg} \cdot \mathrm{ml}^{-1}$, or (3) $250 \mathrm{mg} \cdot \mathrm{ml}^{-1}$ ampoules for use in infusions (recommended dilution 10 $\mathrm{mg} \cdot \mathrm{ml}^{-1}$ ). ${ }^{3}$ Carrying only one form of the drug will reduce inventory costs and may lead to cost savings if wastage is minimized. The vials and ampoules are preservative-free and the company recommends that infusions be changed at least every $24 \mathrm{hr}$ to minimize the risk of microbial contamination. ${ }^{3}$

Departments of Anaesthesia, Pharmacology and Surgery, Dalhousie University, c/o Victoria General Hospital, 1278 Tower Road, Halifax, Nova Scotia, Canada B3H 2 Y9.
The solution is buffered to $\mathrm{pH} 4.5-5.5$. This may be one of the factors leading to the clinical side effect of pain on injection. $^{4}$ The drug is compatible with commonly employed intravenous solutions ${ }^{3}$ but is not compatible with sodium bicarbonate ${ }^{3}$ or furosemide. ${ }^{5}$ Compatibility with atracurium, pancuronium, and vecuronium, ${ }^{6}$ nitroprusside, ${ }^{7}$ aminophylline, bretylium, heparin, and procainamide ${ }^{8}$ has been determined.

In 12 healthy volunteers receiving a bolus injection of esmolol (30-150 mg) the distribution and elimination halftimes could not be calculated due to the rapid disappearance of the drug. ${ }^{9}$ Concentrations decreased below the levels detectable by the assay technique (HPLC with ultraviolet detector) within $10-15$ min. ${ }^{9}$ The authors could not demonstrate a consistent dose vs concentration effect. The only consistent pharmacological effect seen was prolongation of the PR interval and the degree of prolongation did not show a consistent relationship to dose. Peak effect was observed six to ten minutes after injection when the esmolol concentration in plasma was very low (i.e., hysteresis effect). Heart rate was not affected even at the highest dose $(150 \mathrm{mg}$ ).

Sum et al. defined the pharmacokinetic parameters for esmolol following a continuous intravenous infusion at $400 \mathrm{mg} \cdot \mathrm{kg}^{-1} \cdot \mathrm{min}^{-1}$ for two hours. ${ }^{10}$ In their study, clearance approximated to $285 \mathrm{ml} \cdot \mathrm{kg}^{-1} \cdot \mathrm{min}^{-1}$ with a distribution half-time of $2 \mathrm{~min}$ and elimination half-time of $9 \mathrm{~min}$. Volume of distribution was $3.4 \mathrm{~L} \cdot \mathrm{kg}^{-1}$. Metabolism of the drug occurs in the blood by plasma esterases ${ }^{11}$ and is independent of liver ${ }^{12}$ or renal ${ }^{13}$ function. Less than $1 \%$ of the drug is excreted unchanged in the urine and $73 \%$ of the drug is recovered as the inactive $(1 / 1500$ as potent as esmolol) ${ }^{14}$ acid metabolite ASL-8123. ${ }^{15}$ The elimination half-time for the metabolite was prolonged substantially (4.1 to 42 hours) in patients in renal failure. ${ }^{15}$ Approximately $23 \%$ of the drug is removed during dialysis. ${ }^{15}$ These pharmacokinetic parameters indicate that esmolol is a short-acting drug with rapid onset-offset of activity, even following prolonged infusions. This feature is unique among the available $\mathrm{BB}$ agents. For example, among the available intravenous $\mathrm{BB}$, the elimination half-times are $3-5$ hours ${ }^{16}$ suggesting prolonged effect after a bolus dose and a clinically important difference when infusions are 
TABLE Efficacy of esmolol as bolus agent to blunt response to laryngoscopy

\begin{tabular}{|c|c|c|c|c|c|c|}
\hline \multirow[b]{2}{*}{ Study } & \multirow[b]{2}{*}{ Design } & \multirow[b]{2}{*}{$n$} & \multirow[b]{2}{*}{ Comparators } & \multicolumn{3}{|c|}{ Response to esmolol } \\
\hline & & & & $B P$ & $H R$ & Comment \\
\hline Chung et al. ${ }^{1}$ & $\begin{array}{l}\text { Double-blind } \\
\text { Randomized }\end{array}$ & 99 & $\begin{array}{l}\text { Fentanyl } 2 \mu \mathrm{g} \cdot \mathrm{kg}^{-1} \\
\text { Esmolol } 2 \mathrm{mg} \cdot \mathrm{kg}^{-1} \\
\text { Esmolol } 2 \mathrm{mg} \cdot \mathrm{kg}^{-1} \\
\text { Fentanyl } 2 \mu \mathrm{g} \cdot \mathrm{kg}^{-1} \\
\text { Fentanyl } 5 \mu \mathrm{g} \cdot \mathrm{kg}^{-1}\end{array}$ & + & - & $\begin{array}{l}\text { Combination of low-dose fentanyl } \\
+ \text { esmolol superior to either agent } \\
\text { alone. Esmolol alone did not } \\
\text { adequately block heart rate response. }\end{array}$ \\
\hline Miller et $a{ }^{4}{ }^{4}$ & $\begin{array}{l}\text { Multi-centre } \\
\text { Randomized }\end{array}$ & 548 & $\begin{array}{l}\text { Placebo } \\
\text { Esmolol } 100 \mathrm{mg} \\
\text { Esmolol } 200 \mathrm{mg} \\
\text { Fentanyl } 2-3 \mu \mathrm{g} \cdot \mathrm{kg}^{-1} \\
\text { Fentanyl 4-7 } \mu \mathrm{g} \cdot \mathrm{kg}^{-1} \\
\text { + others }\end{array}$ & - & + & $\begin{array}{l}\text { Despite multiple within } \\
\text { centre comparators } \\
\text { efficacy proven. Better } \\
\text { result when combined with } \\
\text { low-dose opioid. Esmolol } \\
100 \mathrm{mg} \text { less side effects. }\end{array}$ \\
\hline Pamass et al. ${ }^{19}$ & $\begin{array}{l}\text { Double-blind } \\
\text { Randomized }\end{array}$ & 30 & $\begin{array}{l}\text { Placebo } \\
\text { Esmolol } 100 \mathrm{mg} \\
\text { Esmolol } 200 \mathrm{mg}\end{array}$ & + & + & $\begin{array}{l}\text { Esmolol } 100 \mathrm{mg} \text { as } \\
\text { effective as } 200 \mathrm{mg}\end{array}$ \\
\hline Ebert et al. ${ }^{20}$ & $\begin{array}{l}\text { Double-blind } \\
\text { Randomized }\end{array}$ & 32 & $\begin{array}{l}\text { Placebo } \\
\text { Esmolol } 100 \mathrm{mg} \\
\text { Esmolol } 200 \mathrm{mg}\end{array}$ & + & + & $\begin{array}{l}\text { Esmolol } 100 \mathrm{mg} \text { as effective as } \\
200 \mathrm{mg} \text { but shorter duration of } \\
\text { effect. Hypotension on induction. }\end{array}$ \\
\hline Mallon et al. ${ }^{21}$ & $\begin{array}{l}\text { Double-blind } \\
\text { Randomized }\end{array}$ & 45 & $\begin{array}{l}\text { Placebo } \\
\text { Esmolol } 100 \mathrm{mg} \\
\text { Esmolol } 200 \mathrm{mg}\end{array}$ & - & + & $\begin{array}{l}\text { Supplementation required } \\
\text { in } 27 \% \text { of esmolol } \\
\text { patients. }\end{array}$ \\
\hline Miller et al. $^{22}$ & $\begin{array}{l}\text { Double-blind } \\
\text { Randomized }\end{array}$ & 45 & $\begin{array}{l}\text { Fentanyl } 2 \mu \mathrm{g} \cdot \mathrm{kg}^{-1} \\
+ \text { placebo } \\
\text { Esmolol } 1.5 \mathrm{mg} \cdot \mathrm{kg}^{-1} \\
\text { Esmolol } 3.0 \mathrm{mg} \cdot \mathrm{kg}^{-1}\end{array}$ & + & + & $\begin{array}{l}\text { Hypotension with higher } \\
\text { dose of esmolol. }\end{array}$ \\
\hline Sheppard $e t a l^{23}$ & $\begin{array}{l}\text { Double-blind } \\
\text { Randomized }\end{array}$ & 45 & $\begin{array}{l}\text { Placebo } \\
\text { Esmolol } 100 \mathrm{mg} \\
\text { Esmolol } 200 \mathrm{mg}\end{array}$ & + & + & $\begin{array}{l}\text { Systolic blood pressure } \\
\text { controlled only at higher } \\
\text { dose. }\end{array}$ \\
\hline Oxorn et al. ${ }^{24}$ & $\begin{array}{l}\text { Double-blind } \\
\text { Randomized }\end{array}$ & 48 & $\begin{array}{l}\text { Placebo } \\
\text { Esmolol } 100 \mathrm{mg} \\
\text { Esmolol } 200 \mathrm{mg}\end{array}$ & - & + & $\begin{array}{l}\text { Hypotension on induction } \\
\text { with the higher dose. }\end{array}$ \\
\hline Helfman et al. ${ }^{25}$ & $\begin{array}{l}\text { Double-blind } \\
\text { Randomized }\end{array}$ & 80 & $\begin{array}{l}\text { Placebo } \\
\text { Lidocaine } 200 \mathrm{mg} \\
\text { Fentanyl } 200 \mu \mathrm{g} \\
\text { Esmolol } 150 \mathrm{mg}\end{array}$ & + & + & $\begin{array}{l}\text { Esmolol more effective } \\
\text { than comparators for HR } \\
\text { control. Timing of doses } \\
\text { an issue. }\end{array}$ \\
\hline
\end{tabular}

prolonged. ${ }^{17}$ Metabolism of propranolol or metoprolol is primarily hepatic and thus affected by changes in liver blood flow/function. ${ }^{16}$

Esmolol has been available world-wide for several years and a large body of knowledge concerning its use both as a bolus injection and/or a continuous infusion is available. Leslie $^{18}$ has suggested that bolus dose esmolol (as utilized in the study by Chung et al. $)^{1}$ might have application by anaesthetists in several areas: (1) to blunt the response to tracheal intubation; $;^{1,4,19-25}(2)$ where there is evidence of intraoperative myocardial ischaemia; ${ }^{26-28}(3)$ where there is a history of coronary artery disease or risk factors; ${ }^{29-31}$ (4) where there is a history of or development of supraventricular tachycardia; ${ }^{32-34}$ (5) hyperthyroidism; ${ }^{35}$ (6) idiopathic subaortic stenosis; (7) tetralogy hypercyanotic spells; (8) electroconvulsive therapy-induced tachycardia and hypertension; ${ }^{36,37}$ (9) hyperdynamic cardiovascular system during a "light" or very short anaesthetic procedure, e.g., outpatients; ${ }^{38}(10)$ tachycardia associated with "anaesthetic breakthrough"; $39-44$ (11) tachycardia associated with vasodilator therapy for controlled hypotension; ${ }^{45}$ (12) anaesthetic emergence hyperdynamic cardiovascular 
activity $;^{46-49}$ and its use in pheochromocytoma and to induce controlled hypotension is reported. . $^{50,51}$

The question of concern to the P\&T Committees, however, is not whether esmolol can be used in any of these circumstances, but whether it should be used - i.e., is more efficacious than other currently available modalities or is more cost effective than currently employed techniques.

Failure to blunt the response to intubation may have disastrous consequences ${ }^{52-54}$ in certain patient populations including those with coronary artery disease, ${ }^{55}$ systemic arterial hypertension, ${ }^{56,57}$ aneurysmal vascular disease, and decreased intracranial compliance..$^{52,58}$

A number of agents has been used to blunt the response to intubation including $i v^{59}$ or topical lidocaine, ${ }^{25,59-61}$ vasodilators ${ }^{62}$ beta adrenergic blockers, ${ }^{63}$ narcotics, ${ }^{1,4,22,25.64-66}$ and inhaled anaesthetics. ${ }^{67}$ All have potential drawbacks which limit their application. Narcotics may produce respiratory depression and truncal rigidity ${ }^{68}$ lidocaine and vasodilators do not reliably obtund the heart rate respons $\mathrm{e}^{25,68,69}$ - although they usually obtund the blood pressure response, propranolol may be effective but its duration of action may extend to several hours and its nonselectivity leads occasionally to bronchospasm. Inhaled anaesthetics may obtund the very responses, e.g., the gag reflex we wish to keep intact prior to the induction of anaesthesia.

Studies examining the role of bolus esmolol in blunting the response to intubation are summarized in the Table. The majority of studies demonstrate that as a bolus dose of $2-3 \mathrm{mg} \cdot \mathrm{kg}^{-1}$, esmolol will blunt the heart rate and to a lesser extent blood pressure response to laryngoscopy but hypotension (although short-lived) post-intubation may occur. Timing of the dose (i.e., two minutes before intubation) is important. ${ }^{1}$ Smaller doses of esmolol, i.e., 1-2 $\mathrm{mg} \cdot \mathrm{kg}^{-1}$ are less effective unless combined with an opioid-fentanyl at $2 \mu \mathrm{g} \cdot \mathrm{kg}^{-1}$ four minutes before intubation as demonstrated in the study by Chung $e t$ al. ${ }^{1}$

Clearly, esmolol alone can be used to blunt the response to tracheal intubation in most patients. In situations where control of the response to intubation is critical, a combination of agents such as the regimen employed by Chung $e t$ al. is recommended. ' Esmolol, especially when combined with fentanyl, is a very reasonable and effective choice. Whether the costs of combining a bolus dose of esmolol with fentanyl to achieve control of the response to laryngoscopy is warranted will be a matter for debate in each of the P\&T Committees.

Clearly esmolol is not just another BB and has many desirable features which makes it use during anaesthesia appealing. I believe that esmolol is both an unique and efficacious agent within the class of BB's available and meets the criteria for inclusion in the hospital formulary.
The decision will be difficult and centred around cost vs efficacy. Anaesthetists must ensure that they are informed about the drug and part of the review process.

\section{Acknowledgement}

The author wishes to acknowledge the assistance of Miss Polly Moores in the preparation of the manuscript.

\section{L'esmolol, un autre bêta-bloqueur?}

Ce mois-ci, dans nos pages, Chung et ses collègues publient les résultats d'une étude randomisée et à double insu ayant pour objectif la prévention de la réaction déclenchée par la laryngoscopie et l'intubation: ils y évaluent l'efficacité d'une injection simple de fentanyl ou d'esmolol, ou l'association des deux drogues à doses réduites. ${ }^{1}$ Leur rapport arrive à point car l'esmolol (Brevibloc) vient tout juste d'obtenir ici l'autorisation nécessaire pour usage clinique : les anesthésistes seront donc sollicités pour l'utiliser dans leur pratique journalière.

Les fonctions d'un comité de pharmacologie consistent à sanctionner, $s^{\prime}$ il se doit, l'avènement de nouveaux agents thérapeutiques en milieu hospitalier, de déterminer s'ils sont réellement efficaces èt de prévenir l'augmentation des coûts. Sous un régime de contraintes budgétaires, la hausse des dépenses causée par l'ajout de nouveaux agents au formulaire peut signifier une réduction des services dans d'autres secteurs hospitaliers, incluant les inconvénients de mises à pieds, fermetures de lits, fermetures de salles d'opération, etc. L'inscription au formulaire d'un nouveau produit ne devient donc justifiable que si elle permet l'accession à une alternative thérapeutique nouvelle et avantageuse, ou encore que le produit à l'étude présente une caractéristique exclusive qui permet de le distinguer d'une famille de médicaments déjà inscrits ayant des caractéristiques identiques. L'esmolol peut être considéré dans cette dernière catégorie puisque sept bêta-bloqueurs sont déjà disponibles au Canada. ${ }^{2}$

L'esmolol s' administre uniquement par la voie veineuse à l'instar de deux autres bêta-bloqueurs : le propanolol et le métoprolol. ${ }^{2}$ Trois présentations sont offertes : 1) des fioles de $10 \mathrm{mg} \cdot \mathrm{ml}^{-1}$ pour injection simple; 2) des ampoules de $100 \mathrm{mg} \cdot \mathrm{ml}^{-1}$ et 3 ) de $250 \mathrm{mg} \cdot \mathrm{ml}^{-1}$ pour la perfusion. On peut réduire les coûts en ne stockant qu' une seule préparation et en minimisant le gaspillage. Les fioles 
et ampoules ne contiennent pas d'agent de conservation et le fabriquant recommande le remplacement d'une perfusion au moins toutes les 24 heures pour diminuer le risque de contamination. ${ }^{3}$

Le $\mathrm{pH}$ de la solution est ramené à 4,5-5,5. Ceci peut expliquer en partie la douleur de l'injection. ${ }^{4} \mathrm{La}$ drogue est compatible avec les solutions intraveineuses d'usage courant mais non avec le bicarbonate de soude ${ }^{3}$ et le furosamide. ${ }^{5}$ On a documenté sa compatibilité avec l'atracurium, le pancuronium, ${ }^{6}$ le nitroprussiate, ${ }^{7}$ l'aminophylline, le brétylium, l'héparine et la procanaïmide. ${ }^{8}$

Chez douze volontaires en bonne santé, après une injection simple d'esmolol (30-150 mg), la disparition rapide du produit n'a pas permis de calculer la demi-vie de distribution et d'élimination. ${ }^{9}$ Les concentrations sont tombées sous le niveau de sensibilité de la méthode de titrage utilisée (HPLS détecteur ultraviolet) en deçà de 10-15 minutes. ${ }^{9}$ Les auteurs n'ont pu démontrer de façon consistante la corrélation réelle entre dose et concentration plasmatique. Le seul effet pharmacologique constant consiste à la prolonger l'intervalle PR à un degré indépendant de la posologie. L'effet maximal est observé ${ }^{6}$ dix minutes après l'injection alors que la concentration plasmatique d'esmolol est à son plus bas niveau (effet d'hystérésis). La fréquence cardiaque n'est pas modifiée même après les plus fortes doses $(150 \mathrm{mg})$.

Sum et ses associés ont déterminé les paramètres pharmacocynétiques de l'esmolol après une perfusion intraveineuse continue de $400 \mu \mathrm{g} \cdot \mathrm{kg}^{-1} \cdot \mathrm{min}^{-1}$ pendant deux heures. ${ }^{10}$ La clairance s'établit à environ 285 $\mathrm{ml} \cdot \mathrm{kg}^{-1} \cdot \mathrm{min}^{-1}$ avec une demi-vie de distribution de deux minutes et d'élimination de neuf minutes. Le volume de distribution se situe à $3,4 \mathrm{~L} \cdot \mathrm{kg}^{-1}$. Le métabolisme relève des estérases plasmatiques ${ }^{11}$ et est indépendant des fonctions hépatique ${ }^{12}$ et rénale. ${ }^{13}$ Moins de $1 \%$ de la drogue est excrétée inchangée dans l'urine et on retrouve $73 \%$ sous forme du métabolite acide ASL-8123 (1,500 moins puissant que l'esmolol.) ${ }^{15}$ La demi-vie du métabolite se prolonge considérablement $(4,1-42$ heures) chez les insuffisants rénaux. ${ }^{15} \mathrm{La}$ dyalise extrait $23 \%$ du produit. Ces données pharmacocynétiques démontrent que l'esmolol est un médicament à courte durée d'action, à installation et disparition rapides même après des perfusions prolongées. Cette caractéristique est unique parmi les bêta-bloqueurs mis à notre disposition. Pour ces derniers, la demi-vie d'élimination est de 3-5 heures, ${ }^{16}$ ce qui suggère un effet prolongé après un bolus et une différence cliniquement importante avec l'esmolol pour les perfusions de longue durée. ${ }^{17}$ Le métabolisme du propanolol ou du métoprolol est surtout hépatique, en conséquence affecté par les modifications du débit sanguin et de la fonction hépatiques.
On utilise l'esmolol partout dans le monde depuis plusieurs années et on connaît très bien son mode d'emploi en bolus ou en perfusion. Leslie ${ }^{18}$ croit que l'esmolol en bolus (tel qu'utilisé dans l'étude de Chung et coll. ') pourrait trouver des applications dans plusieurs interventions anesthésiques : 1) pour atténuer la réponse à l'intubation endotrachéale ;,4,19-25 2) durant les épisodes d'ischémie myocardique peropératoire; 3) chez les patients présentant des antécédents de maladie coronaire ou des facteurs de risque apparentés $;^{29-31}$ 4) lorsque le patient a une histoire de tachycardie supraventriculaire ;2-34 5) dans l'hyperthyroïdie $;^{35} 6$ ) dans la sténose sous-aortique idiopathique ; 7) contre les attaques cyanogènes de la tétralogie de Fallot ; 8) contre la tachycardie et l'hypertension consécutives à la sismothérapie ; ${ }^{36,37} 9$ ) pour stabiliser un système vasculaire hyperdynamique pendant une anesthésie légère ou une intervention courte, spécialement chez le patient ambulatoire ; ${ }^{38} 10$ ) lors de tachycardie associée à un allègement de l'anesthésie peropératoire $;^{39-44} 11$ ) contre la tachycardie accompagnant l'hypotension contrôlée réalisée avec des vasodilatateurs ; ${ }^{45} 12$ ) à l'émergence d'une l'anesthésie s'accompagnant d'hyperdynamisme cardiovasculaire. ${ }^{6-49}$ On rapporte aussi son utilisation pour la prise en charge du phéochromocytome et pour l'induction de l'hypotension délibérée. ${ }^{50,51}$ L'incapacité d'atténuer la réponse à l'intubation peut s'avérer désastreuse $\mathrm{s}^{52-54}$ pour certains patients dont les coronariens, ${ }^{52-54}$ les hypertendus, ${ }^{56-57}$ les porteurs d'anévrysme et les malades dont la compliance intracrânienne est diminuée. ${ }^{52-58}$

Les anesthésistes utilisent plusieurs produits pour atténuer la réponse à l'intubation dont la lidocaïne topique ou $i v,{ }^{25,59-61}$ les vasodilatateurs, les bêta-bloqueurs, ${ }^{63}$ les opiacés ${ }^{1,4,22,25,64-66}$ et les agents d'inhalation. Tous ont des inconvénients potentiels qui limitent leur application. Les opiacés peuvent produire une dépression respiratoire, de la rigidité tronculaire $;^{68}$ la lidocaïne et les vasodilatateurs sont plus ou moins fiables pour le contrôle de la tachycardie, ${ }^{25,68,69}$ bien qu'ils abolissent ordinairement l'hypertension; le propanolol est la plupart du temps efficace mais son activité peut se prolonger pendant de longues heures et son manque de sélectivité peut provoquer du bronchospasme ; les agents d'inhalation peuvent émousser certaines réactions que nous désirons conserver intactes comme le réflexe pharyngé pendant l'induction de l'anesthésie.

Les études concernant la suppression de la réponse à l'intubation par un bolus d'esmolol sont résumées au tableau. La majorité des observations montrent qu'un bolus de $2-3 \mu \mathrm{g} \cdot \mathrm{kg}^{-1}$ d'esmolol prévient la tachycardie et à un degré moindre, l'augmentation de la pression artrielle, de la laryngoscopie mais une brève période d'hypotension 
TABLEAU Efficacité de l'esmolol en bolus sur la réponse à la laryngoscope

\begin{tabular}{|c|c|c|c|c|c|c|}
\hline \multirow[b]{2}{*}{ Auteurs } & \multirow[b]{2}{*}{ Modele } & \multirow[b]{2}{*}{$n$} & \multirow[b]{2}{*}{ Comparaisons } & \multicolumn{3}{|c|}{ Réponse à l'esmolol } \\
\hline & & & & T.A. & $F c$ & Commentaire \\
\hline Chung et coll. ${ }^{1}$ & $\begin{array}{l}\text { Double insu et } \\
\text { randomisation }\end{array}$ & 99 & $\begin{array}{l}\text { Fentanyl } 2 \mu \mathrm{g} \cdot \mathrm{kg}^{-1} \\
\text { Esmolol } 2 \mathrm{mg} \cdot \mathrm{kg}^{-1} \\
\text { Esmolol } 2 \mathrm{mg} \cdot \mathrm{kg}^{-1} \\
\text { Fentanyl } 2 \mu \mathrm{g} \cdot \mathrm{kg}^{-1} \\
\text { Fentanyl } 5 \mu \mathrm{g} \cdot \mathrm{kg}^{-1}\end{array}$ & + & - & $\begin{array}{l}\text { L'association de fentanyl et } \\
\text { d'esmolol à faibles doses } \\
\text { supérieure à chacun des } \\
\text { agents seuls. Bloc inadéquat } \\
\text { de la réponse cardiaque }\end{array}$ \\
\hline Miller et coll. ${ }^{4}$ & $\begin{array}{l}\text { Multi-centrique } \\
\text { et randomisation }\end{array}$ & 548 & $\begin{array}{l}\text { Placebo } \\
\text { Esmolol } 100 \mathrm{mg} \\
\text { Esmolol } 200 \mathrm{mg} \\
\text { Fentanyl 2-3 } \mu \mathrm{g} \cdot \mathrm{kg}^{-1} \\
\text { Fentanyl 4-7 } \mathrm{\mu g} \cdot \mathrm{kg}^{-1} \\
+ \text { autres }\end{array}$ & - & + & $\begin{array}{l}\text { Efficacité prouvée malgré } \\
\text { multiples comparaisons. } \\
\text { Résultats supérieurs avec } \\
\text { opiacés à petites doses. } \\
\text { Esmolol } 100 \mathrm{mg} \text { a moins } \\
\text { d'effets secondaires }\end{array}$ \\
\hline Parnass et coll. ${ }^{19}$ & $\begin{array}{l}\text { Double insu et } \\
\text { randomisation }\end{array}$ & 30 & $\begin{array}{l}\text { Placebo } \\
\text { Esmolol } 100 \mathrm{mg} \\
\text { Esmolol } 200 \mathrm{mg}\end{array}$ & + & + & $\begin{array}{l}\text { Esmolol } 100 \mathrm{mg} \text { aussi } \\
\text { efficace que } 200 \mathrm{mg}\end{array}$ \\
\hline Ebert et coll. ${ }^{20}$ & $\begin{array}{l}\text { Double insu et } \\
\text { randomisation }\end{array}$ & 32 & $\begin{array}{l}\text { Placebo } \\
\text { Esmolol } 100 \mathrm{mg} \\
\text { Esmolol } 200 \mathrm{mg}\end{array}$ & + & + & $\begin{array}{l}\text { Esmolol } 100 \mathrm{mg} \text { aussi } \\
\text { efficace que } 200 \mathrm{mg} \text { mais } \\
\text { effet de plus courte durée. } \\
\text { Hypotension à l'induction }\end{array}$ \\
\hline Mallon et coll. ${ }^{21}$ & $\begin{array}{l}\text { Double insu et } \\
\text { randomisation }\end{array}$ & 45 & $\begin{array}{l}\text { Placebo } \\
\text { Esmolol } 100 \mathrm{mg} \\
\text { Esmolol } 200 \mathrm{mg}\end{array}$ & - & + & $\begin{array}{l}\text { Supplémentation requise } \\
\text { dans } 27 \% \text { des cas d'esmolol }\end{array}$ \\
\hline Miller et coll. ${ }^{22}$ & $\begin{array}{l}\text { Double insu et } \\
\text { randomisation }\end{array}$ & 45 & $\begin{array}{l}\text { Fentanyl } 2 \mu \mathrm{g} \cdot \mathrm{kg}^{-1} \\
\text { + placebo } \\
\text { Esmolol } 1.5 \mathrm{mg} \cdot \mathrm{kg}^{-1} \\
\text { Esmolol } 3.0 \mathrm{mg} \cdot \mathrm{kg}^{-1}\end{array}$ & + & + & $\begin{array}{l}\text { Hypotension avec les plus } \\
\text { hautes doses d'esmolol }\end{array}$ \\
\hline Sheppard et coll. ${ }^{23}$ & $\begin{array}{l}\text { Double insu et } \\
\text { randomisation }\end{array}$ & 45 & $\begin{array}{l}\text { Placebo } \\
\text { Esmolol } 100 \mathrm{mg} \\
\text { Esmolol } 200 \mathrm{mg}\end{array}$ & + & + & $\begin{array}{l}\text { La pression systolique n'est } \\
\text { contrôlée qu'avec la plus } \\
\text { forte dose }\end{array}$ \\
\hline Oxom et coll. ${ }^{24}$ & $\begin{array}{l}\text { Double insu et } \\
\text { randomisation }\end{array}$ & 48 & $\begin{array}{l}\text { Placebo } \\
\text { Esmolol } 100 \mathrm{mg} \\
\text { Esmolol } 200 \mathrm{mg}\end{array}$ & - & + & $\begin{array}{l}\text { Hypotension a l'induction } \\
\text { avec la dose la plus forte }\end{array}$ \\
\hline Helfman et coll. ${ }^{25}$ & $\begin{array}{l}\text { Double insu et } \\
\text { randomisation }\end{array}$ & 80 & $\begin{array}{l}\text { Placebo } \\
\text { Lidocaine } 200 \mathrm{mg} \\
\text { Fentanyl } 200 \mu \mathrm{g} \\
\text { Esmolol } 150 \mathrm{mg}\end{array}$ & + & + & $\begin{array}{l}\text { Esmolol plus efficace que les } \\
\text { autres agents pour le contrôle } \\
\text { de Fc. La synchronisation est } \\
\text { importante }\end{array}$ \\
\hline
\end{tabular}

peut survenir après l'intubation. La synchronisation de la dose (deux minutes avant l'intubation) est importante. Des doses moindres d'esmolol sont efficaces lorsqu'elles sont associées à un morphinique comme le fentanyl $2 \mu \mathrm{g} \cdot \mathrm{kg}^{-1}$, quatre minutes avant l'intubation comme le démontrent Chung et ses collaborateurs.'

De toute évidence, l'esmolol peut être utilisé pour atténuer la réaction à l'intubation chez la plupart des patients. Dans les situations où le contrôle de cette réponse est critique, une association d'agents comme le suggère
Chung et coll. est recommandable, ${ }^{1}$ spécialement le fentanyl. La question du coût de cette association esmololfentanyl injectée en bolus deviendra un bon sujet de discussion pour les membres du comité de pharmacologie.

L'esmolol n'a rien d'un bêta-bloqueur ordinaire et présente plusieurs caractéristiques désirables et attrayantes pour l'anesthésiste. Je crois que l'esmolol est à la fois unique et efficace dans sa classe et qu'il rencontre les critères d'inscription au formulaire de l'hôpital. Cette décision reposera sur l'analyse comparative des coûts et de 
l'efficacité. Les anesthésistes se doivent de bien connaître le médicament et ils doivent participer activement au processus d'évaluation.

\section{References}

1 Chung KS, Sinatra RS, Halevy JD, et al. A comparison of fentanyl, esmolol, and their combination as anesthetic adjuncts during rapid sequence induction. Can J Anaesth 1992; 39: 000-00.

2 Compendium of Pharmaceuticals and Specialties: 26th ed. Canadian Pharmaceutical Association; Ed. CME Krogh, 1991, Toronto, pp 1-1336.

3 Product Monograph - Brevibloc® (esmolol hydrochloride), DuPont Pharma, DuPont Merck Pharma Inc., Mississauga, Ontario, 1992.

4 Miller DR, Martineau RJ, Wynands JE, et al. Bolus administration of esmolol for controlling the haemodynamic response to tracheal intubation: the Canadian multicentre trial. Can J Anaesth 1991; 38: 849-58.

5 Thompson DE, Thompson GD. Visual compatibility of esmolol hydrochloride and furosemide in 5\% dextrose or 0.9\% sodium chloride injections. Am J Hosp Pharm 1987; 44: 2740.

6 Savitsky ME. Visual compatibility of neuromuscular blocking agents with various injectable drugs during simulated Y-site injection. Am J Hosp Pharm 1990; 47 : 820-1.

7 Karnatz NN, Wong J, Baaske DM, et al. Stability of esmolol hydrochloride and sodium nitroprusside in intravenous admixtures. Am J Hosp Pharm 1989; 46: 101-4.

8 Schaaf $L$, Robinson DH, Vogel GJ, et al. Stability of esmolol hydrochloride in the presence of aminophylline, bretylium tosylate, heparin sodium, and procainamide hydrochloride. Am J Hosp Pharm 1990; 47: 1567-71.

9 Sintetos AL, Hulse J, Pritchett ELC. Pharmacokinetics and pharmacodynamics of esmolol administered as an intravenous bolus. Clin Pharmacol Ther 1987; 41: 112-7.

10 Sum CY, Yacobi A, Kartzinel R, et al. Kinetics of esmolol, an ultra-short-acting beta blocker, and of its major metabolite. Clin Pharmacol Ther 1983; 34: 427-34.

11 Quon CY, Stampfli $H F$. Biochemical properties of blood esmolol esterase. Drug Metab Dispos 1985; 13: $420-4$.

12 Buchi KN, Rollins DE, Tolman KG, et al. Pharmacokinetics of esmolol in hepatic disease. J Clin Pharmacol 1987; 27: $880-4$

13 Flaherty JF, Wong B, LaFollette G, et al. Pharmacokinetics of esmolol and ASL-8123 in renal failure. Clin Pharmacol Ther 1989; 45: 321-7.

14 Shaffer JE, Quen CY, Georezynski RJ. Beta adrenoreceptor antagonist potency and pharmacodynamics of ASL-8123, the primary acid metabolite of esmolol. J Cardiovasc Pharmacol 1988; 11: 187-92.

15 Achari R, Drissel D, Matier WL, et al. Metabolism and urinary excretion of esmolol in humans. J Clin Pharmacol 1986; 26: 44-7.

16 Stoelting RK. Pharmacology and Physiology in Anesthetic Practice, 2nd ed., Philadelphia, 1991, JP Lippincott, pp 295-310.

17 Hughes MA, Glass PSA, Jacobs JR. Context-sensitive half-time in multicompartment pharmacokinetic models for intravenous drugs. Anesthesiology 1992; 76: 334-41.

18 Leslie JB. Bolus dose esmolol for anesthesia: is there a need? J Clin Anesth 1990; 2: 215-20.

19 Parnass SM, Rothenberg DM, Kerchberger JP, Ivankovich $A D$. A single bolus dose of esmolol in the prevention of intubation-induced tachycardia and hypertension in an ambulatory surgery unit. J Clin Anesth 1990; 2: 232-7.

20 Ebert TJ, Bernstein JS, Stowe DF, Roerig D, Kampine JP. Attenuation of hemodynamic responses to rapid sequence induction and intubation in healthy patients with a single bolus of esmolol. J Clin Anesth 1990; 2: 243-52.

21 Mallon JS, Hew E, Wald R, et al. Bolus doses of esmolol for the prevention of postintubation hypertension and tachycardia. J Cardiothorac Anesth 1990; 4 (Suppl 2): 27-30.

22 Miller DR, Martineau RJ, Hull KA, et al. Bolus administration of esmolol for controlling the hemodynamic response to laryngoscopy and intubation: efficacy and effects on myocardial performance. J Cardiothorac Anesth 1990; 4 (Suppl 2): 31-6.

23 Sheppard S, Eagle CJ, Strunin L. A bolus dose of esmolol attenuates tachycardia and hypertension after tracheal intubation. Can J Anaesth 1990; 37: 202-5.

24 Oxorn D, Knox JWD, Hill J. Bolus doses of esmolol for the prevention of perioperative hypertension and tachycardia. Can J Anaesth 1990; 37: 206-9.

25 Helfman SM, Gold MI, DeLisser EA, et al. Which drug prevents tachycardia and hypertension associated with tracheal intubation: lidocaine, fentanyl, or esmolol? Anesth Analg 1991; 72: 482-6.

26 Miller DR, Martineau RJ. Bolus administration of esmolol for the treatment of intraoperative myocardial ischaemia. Can J Anaesth 1989; 36: 593-7.

27 Kanitz DD, Ebert TJ, Kampine JP. Intraoperative use of bolus doses of esmolol to treat tachycardia. J Clin Anesth 1990; 2: 238-42.

28 Menkhaus PG, Reves JG, Kissin I, et al. Cardiovascular effects of esmolol in anesthetized humans. Anesth Analg 1985; 64: 327-34.

29 Kirshenbaum JM, Kloner RA, Antman EM, et al. Use of an ultra short-acting $\beta$-blocker in patients with acute myocardial ischemia. Circulation 1985; 72: 873-80.

30 Labovitz AJ, Barth C, Castello R, et al. Attenuation of myocardial ischemia during coronary occlusion by ultrashort-acting beta adrenergic blockade. Am Heart J 1991; 121: $1347-52$. 
31 Wallis DE, Wedel VA, Scanlon PJ, et al. Effect of esmolol on the ventricular fibrillation threshold. Pharmacology 1988; 36: 9-15.

32 The Esmolol Multicenter Study Research Group: efficacy and safety of esmolol vs propranolol in the treatment of supraventricular tachyarrhythmias: a multicenter doubleblind clinical trial. Am Heart J 1985; 110: 913-22.

33 Gray RJ, Bateman TM, Czer LS, et al. Esmolol: a new ultrashort-acting beta-adrenergic blocking agent for rapid control of heart rate in postoperative supraventricular tachyarrhythmias. J Am Coll Cardiol 1985; 5: 1451-6.

34 Tuman $K J$, McCarthy RJ, Wong CA, et al. Comparative effects of esmolol and verapamil in a model of a supraventricular tachydysrhythmia. Anesthesiology 1990; 73: 467-73.

35 Brunette $D D$, Rothong $C$. Emergency department management of thyrotoxic crisis with esmolol. Am J Emerg Med 1991; 9: 232-4.

36 Howie MB, Black HA, Zvara D, et al. Esmolol reduces autonomic hypersensitivity and length of seizures induced by electroconvulsive therapy. Anesth Analg 1990; 71(4): 384-8.

37 Kovac AL, Goto H, Pardo MP, et al. Comparison of two esmolol bolus doses on the haemodynamic response and seizure duration during electroconvulsive therapy. Can J Anaesth 1991; 38: 204-9.

38 Sandler AN, Leitch LF, Badner NH, et al. Esmolol compared with placebo in preventing increases in heart rate and blood pressure during rigid bronchoscopy. J Cardiothorac Anesth 1990; 4 (Suppl 2): 44-50.

39 Nicholson SC, Jobes DR, Quinlan JJ. Cardiovascular effects of esmolol in patients anesthetised with sufentanilpancuronium for myocardial revascularisation. J Cardiothorac Anesth 1990; 4 (Suppl 2): 55-8.

40 Kataja JHK, Kaukinen S, Viinamäki OVK, et al. Esmolol for treatment of hypertension and tachycardia in patients during and after abdominal aortic surgery. $J$ Cardiothorac Anesth 1990; 4 (Suppl 2): 37-43.

41 Girard D, Shulman BJ, Thys DM, et al. The safety and efficacy of esmolol during myocardial revascularization. Anesthesiology 1986; 65: 157-64.

42 Ramsay JG. Esmolol (Editorial). Can J Anaesth 1991; 38: 155-8.

43 Newsome LR, Roth JV, Hug CC Jr, et al. Esmolol attenuates hemodynamic responses during fentanyl-pancuronium anesthesia for aortocoronary bypass surgery. Anesth Analg 1986; 65: 451-6.

44 Harrison L, Ralley FE, Wynands JE, et al. The role of an ultra short-acting adrenergic blocker (esmolol) in patients undergoing coronary artery bypass surgery. Anesthesiology $1987 ; 66: 413-8$.

45 Edmondson $R$, DelValle $O$, Shah N, et al. Esmolol for potentiation of nitroprusside-induced hypotension: impact on the cardiovascular, adrenergic, and renin-angiotensin systems in man. Anaesth Analg 1989; 69: 202-6.

46 Kataria $B$, Dubois $M$, Lea $D$, et al. Evaluation of intravenous esmolol for treatment of postoperative hypertension. J Cardiothorac Anesth 1990; 4 (Suppl 2): 13-6.

47 Gray RJ, Bateman TM, Czer LSC, et al. Comparison of esmolol and nitroprusside for acute post-cardiac surgical hypertension. Am J Cardiol 1987; 59: 887-91.

48 Gibson BE, Black $S$, Maass $L$, et al. Esmolol for the control of hypertension after neurologic surgery. Clin Pharmacol Ther 1988; 44: 650-3.

49 Muzzi DA, Black S, Losasso TJ, et al. Labetalol and esmolol in the control of hypertension after intracranial surgery. Anesth Analg 1990; 70: 68-71

50 Mihm FG, Sandhu JS, Brown MD, et al. Short-acting beta-adrenergic blockade as initial drug therapy in pheochromocytoma. Crit Care Med 1990; 18: 673-4

51 Ornstein E, Young WL, Ostapkovich $N$, et al. Deliberate hypotension in patients with intracranial arteriovenous malformations: esmolol compared with isoflurane and sodium nitroprusside. Anesth Analg 1991; 72: 639-44.

52 Fox EJ, Sklar GS, Hill CH, et al. Complications related to the pressor response to endotracheal intubation. Anesthesiology $1977 ; 47$ : 524-5.

53 S $\phi$ rensen $M B$, Jacobsen E. Pulmonary hemodynamics during induction of anesthesia. Anesthesiology 1977; 46: 246-51.

54 Tomori $Z$, Widdicombe JG. Muscular, bronchomotor and cardiovascular reflexes elicited by mechanical stimulation of the respiratory tract. J Physiol 1969; 200: 25-49.

55 Roy WL, Edelist G, Gilbert B. Myocardial ischemia during noncardiac surgical procedures in patients with coronary artery disease. Anesthesiology 1979; 51: 393-7.

56 Prys-Roberts C, Greene LT, Meloche R, et al. Studies of anaesthesia in relation to hypertension II: haemodynamic consequences of induction and endotracheal intubation. $\mathrm{Br}$ J Anaesth 1971; 43: 531-46.

57 Shepard LC, Gelman S, Reeves JG, et al. Humoral response of hypertensive patients to laryngoscopy. Anesth Analg 1981; 60: 276-7.

58 Shapiro HM, Wyte SR, Harris AB, Galindo A. Acute intraoperative intracranial hypertension in neurosurgical patients: mechanical and pharmacologic factors. Anesthesiology 1972; 37: 399-405.

59 Stoelting $R K$. Blood pressure and heart rate changes during short-duration laryngoscopy for tracheal intubation: Influence of viscous or intravenous lidocaine. Anesth Analg 1978; 57: 197-9.

60 Denlinger JK, Ellison N, Ominsky AJ. Effects of intratracheal lidocaine on circulatory responses to tracheal intubation. Anesthesiology 1974; 41: 409-12.

61 Hamill JF, Bedford RF, Weaver DC, et al. Lidocaine before endotracheal intubation: intravenous or 
laryngotracheal? Anesthesiology 1981; 55: 578-81.

62 Stoelting $R K$. Attenuation of blood pressure response to laryngoscopy and tracheal intubation with sodium nitroprusside. Anesth Analg 1979; 58: 116-9.

63 Safwat AM, Reitan JA, Misle GR, et al. Use of propranolol to control rate-pressure product during cardiac anesthesia. Anesth Analg 1981; 60: 732-5.

64 Martin DE, Rosenberg H, Aukburg SJ, et al. Low-dose fentanyl blunts circulatory responses to tracheal intubation. Anesth Analg 1982; 61: 680-4.

65 lyer $V$, Russell $W$. Induction using fentanyl to suppress the intubation response in the cardiac patient: what is the optimal dose? Anaesth Intens Care 1988; 16: 411-7.

66 Dahlgren $N$, Messeter $K$. Treatment of stress response to laryngoscopy and intubation with fentanyl. Anaesthesia 1981; 36: 1022-6.

67 King B, Harris L, Greifenstein F, et al. Reflex circulatory responses to direct laryngoscopy and tracheal intubation performed during general anesthesia. Anesthesiology 1951; 12: 556-66.

68 Splinter WM, Cervenko $F$. Haemodynamic responses to laryngoscopy and tracheal intubation in geriatric patients: effects of fentanyl, lidocaine and thiopentone. Can J Anaesth 1989; 36: 370-6.

69 Chraemmer-Jørgensen B, Høilund-Carlsen PF, Marving $J$, et al. Lack of effect of intravenous lidocaine on hemodynamic responses to rapid sequence induction of general anesthesia: a double-blind controlled clinical trial. Anesth Analg 1986; 65: 1037-41. 